\title{
LODO DE ESGOTO TRATADO NA COMPOSIÇÃO DE SUBSTRATO PARA PRODUÇÃO DE MUDAS DE Lafoensia glyptocarpa
}

\author{
David Pessanha Siqueira ${ }^{*}$, Giovanna Campos Mamede Weiss de Carvalho ${ }^{1}$; Deborah Guerra Barroso²; Cláudio \\ Roberto Marciano ${ }^{3}$
1* Universidade Estadual do Norte Fluminense Darcy Ribeiro, Programa de Pós Graduação em Produção Vegetal, Campos dos Goytacazes, Rio de Janeiro, Brasil - dps@pq.uenf.br*; giovannacampos85@yahoo.com.br
${ }^{2}$ Universidade Estadual do Norte Fluminense Darcy Ribeiro, Laboratório de Fitotecnia, Campos dos Goytacazes, Rio de Janeiro, Brasil - deborahbarroso@gmail.com
${ }^{3}$ Universidade Estadual do Norte Fluminense Darcy Ribeiro, Laboratório de Solos, Campos dos Goytacazes, Rio de Janeiro, Brasil - marciano@uenf.br

Recebido para publicação: 12/10/2017 - Aceito para publicação: 23/02/2018

\begin{abstract}
Resumo
Buscando-se alternativas para a melhor destinação deste resíduo e para o suprimento da crescente demanda por mudas nativas, este trabalho teve por objetivo avaliar o uso do lodo tratado na composição de substrato para produção de mudas de mirindiba-rosa. Foram avaliadas características biométricas e fisiológicas das mudas e a qualidade do torrão formado. Para tratamento do lodo, foi feita aplicação de cal a $15 \%$ do peso seco do resíduo, sendo testadas as proporções de lodo de esgoto tratado de 25,50 e $75 \%$, associadas ao substrato comercial, e $100 \%$ de lodo de esgoto tratado. O tratamento com $100 \%$ de substrato comercial foi considerado como controle. O experimento foi conduzido em delineamento inteiramente casualizado (DIC), os dados foram submetidos a ANOVA, e as diferenças foram comparadas pelo teste de Tukey a 5\%. Os melhores resultados foram observados nas mudas produzidas em substratos com lodo de esgoto tratado em relação ao substrato comercial puro. Recomenda-se, para essa espécie, proporções entre 25 e $100 \%$ de lodo de esgoto tratado em substrato comercial.

Palavras-chave: Resíduo urbano, mirindiba-rosa, viveiro florestal, qualidade do torrão.
\end{abstract}

\begin{abstract}
Treated sewage sludge in substrate composition for the production of Lafoensia glyptocarpa seedlings In order to search for alternatives of better allocation of sewage sludge and supply of the increasing demand for native tree seedlings, this study aimed to assess the treated sewage sludge in substrate composition of mirindiba-rosa seedlings production. Biometrical and physiological characteristics of the seedlings and the clod quality were assessed. For treating the sludge, a lime application was done at $15 \%$ of residue dry weight. The following proportions of treated sewage sludge were tested: 25,50 , and $75 \%$, blended with commercial substrate, and $100 \%$ of treated sewage sludge. The treatment with $100 \%$ of commercial substrate was the control. The study was conducted in a completely randomized design (DIC), data were subjected to ANOVA, and the differences were compared by the Tukey test at 5\%. The best results were observed for the seedlings produced in substrates with treated sewage sludge in comparison to pure commercial substrate. Proportions between 25 and $100 \%$ of treated sewage sludge on commercial substrate are recommended for this species.

Keywords: Urban waste, mirindiba-rosa, forest nursery, clod quality.
\end{abstract}

\section{INTRODUÇÃO}

A mirindiba-rosa (Lafoensia glyptocarpa) é uma espécie nativa da Mata Atlântica, pertencente à família Lythraceae, considerada de rápido crescimento. É recomendada para plantios mistos, destinados à recuperação de áreas degradadas e de preservação permanente, bem como para utilização na arborização devido à sua rusticidade e belas flores (LORENZI, 2002). Atinge de 15 a 25 metros de altura e 40 a $60 \mathrm{~cm}$ de diâmetro, com floração podendo ocorrer de julho a agosto (CARVALHO, 2003). Foi observado que árvores adultas, isoladas em via pública, são capazes de atenuar $64 \%$ da radiação solar, contribuindo para o conforto térmico (ABREU HARBICH et al., 2015).

A constante exploração das florestas nativas, seja pela expansão agrícola, pela pecuária extensiva ou pelo aproveitamento direto da madeira, muitas vezes de forma ilegal, acarreta diminuição da cobertura florestal, provocando degradação e desequilíbrio ambiental (CALDEIRA et al., 2013). Esses fatores contribuem para o aumento da demanda de mudas nativas, que deverão ser de boa qualidade, para adequada recuperação das áreas degradadas, reflorestamento ou soluções ambientais (DELARMELINA et al., 2014).

A composição do substrato é um fator que influencia a qualidade das mudas formadas, devendo fornecer suporte físico ao sistema radicular e condições para suprir adequadamente a demanda hídrica e nutricional da muda. Caldeira et al. (2012),

FLORESTA, Curitiba, PR, v. 48, n. 2, p. 277-284, abr/jun. 2018.

Siqueira. D. P. et.al.

ISSN eletrônico 1982-4688

DOI: $10.5380 /$ rf.v48 i2.55795 
entre vários outros autores, recomendam a mistura de materiais para composição do substrato. De modo geral, é difícil encontrar um material isolado que satisfaça todas as condições necessárias às diferentes espécies florestais.

O lodo de esgoto é um resíduo de caráter orgânico, semissólido e com variáveis teores de componentes inorgânicos que, em sua grande maioria, é destinado aos aterros sanitários, procedimento complexo e oneroso (GOMES et al., 2013). Assim, a destinação agrícola e florestal do lodo se destaca, devido a riqueza nutricional, alto teor de matéria orgânica e elevada retenção de água, características de grande interesse agrícola (CALDEIRA et al., 2013; SANTOS et al., 2014; NOBREGA et al., 2017).

Quando utilizado na formulação de substratos para produção de mudas, deve-se ter cautela com relação ao teor do lodo na composição total do substrato, tanto por suas características físicas quanto químicas, devido à possibilidade de possuírem elevadas concentrações de alguns nutrientes e substâncias inorgânicas prejudiciais às plantas, como os metais pesados (GOMES et al., 2013; ROCHA et al, 2013; TRAZZI et al., 2014).

Pesquisas relacionadas ao efeito de diferentes composições de substrato sobre a qualidade de mudas formadas são constantes e atuais, e busca-se integrar os preceitos da sustentabilidade, oferecer alternativas ao produtor e proporcionar redução de tempo e custos do processo produtivo. Assim, este estudo teve como objetivo avaliar o uso de lodo de esgoto tratado na composição de substrato para produção de mudas de Lafoensia glyptocarpa.

\section{MATERIAL E MÉTODOS}

O experimento foi conduzido em casa de vegetação, localizada na Unidade de Apoio à Pesquisa (UAP), pertencente à Universidade Estadual do Norte Fluminense (UENF), no município de Campos dos Goytacazes, RJ. Foi instalado na casa de vegetação o medidor de temperatura e umidade relativa (Extech - RHT10) para monitoramento durante o período experimental, observando-se temperatura e umidade relativa médias de $27,1^{\circ} \mathrm{C}$ e $78,7 \%$, respectivamente.

O experimento foi conduzido no período de julho a novembro de 2015 em delineamento inteiramente casualizado (DIC), com cinco repetições e seis mudas por parcela.

A espécie utilizada foi a mirindiba-rosa (Lafoensia glyptocarpa), semeada em bandejas plásticas, contendo substrato comercial (SC). Após 10 dias, as plântulas foram repicadas para tubetes de $180 \mathrm{~cm}^{3}$, preenchidos com as seguintes proporções de lodo de esgoto tratado (LET): 25, 50 e 75\%, associadas ao SC, e um tratamento composto por $100 \%$ de LET. Considerou-se como testemunha o tratamento utilizando o SC puro. O substrato comercial utilizado foi o Basaplant Florestal.

O lodo de esgoto utilizado foi doado pela empresa "Águas do Paraíba", prestadora de serviços do Município de Campos dos Goytacazes (RJ), e produzido na estação de tratamento da Chatuba, cujos teores de nutrientes e metais pesados estão descritos na Tabela 1. Os valores observados no lote foram comparados aos limites preconizados pela legislação vigente para utilização agrícola e florestal (CONSELHO NACIONAL DO MEIO AMBIENTE, 2006). O lote do lodo estava apto para tal destinação. Para higienização e estabilização do lodo, foi adicionada cal (CONSELHO NACIONAL DO MEIO AMBIENTE, 2006) a 15\% do peso seco. Apesar do lote do lodo estar apto para destinação agrícola, foram observadas manchas foliares nas mudas, mais acentuadas nas maiores proporções de lodo (50, 75 e 100\%), que possivelmente foram ocasionadas pela presença dos metais pesados no resíduo, além dos elevados teores de nutrientes.

Tabela 1. Atributos químicos do lodo de esgoto caleado utilizado na composição de substratos para produção de mudas de Lafoensia glyptocarpa.

Table 1. Chemical attributes of sewage sludge treated with lime in substrate composition for the production of Lafoensia glyptocarpa seedlings.

\begin{tabular}{|c|c|c|c|c|c|c|c|c|c|c|}
\hline Umidade & pH & $\mathbf{N}$ & $\mathbf{P}$ & $\mathbf{K}$ & $\mathbf{C a}$ & Mg & $\mathbf{S}$ & $\mathbf{C}$ & Fe & $\mathbf{C u}$ \\
\hline$\%$ & \multicolumn{10}{|c|}{ - } \\
\hline 38,2 & 8,0 & 15,75 & 5,84 & 1,22 & 111,70 & 3,07 & 12,53 & 129,6 & 40,3 & 306 \\
\hline $\mathrm{Na}$ & $\mathrm{Zn}$ & $\mathrm{Mn}$ & B & $\mathrm{Al}$ & $\mathrm{Ba}$ & $\mathrm{Cd}$ & $\mathrm{Cr}$ & $\mathrm{Ni}$ & $\mathrm{Pb}$ & $\mathrm{Sr}$ \\
\hline \multicolumn{11}{|c|}{ 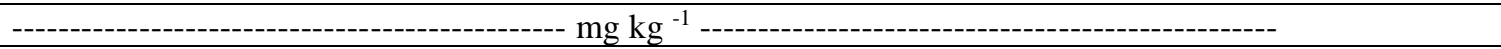 } \\
\hline 403,52 & 588 & 244 & 20,06 & 5457,28 & 102,60 & 1,95 & 19,92 & 7,58 & 14,77 & 202,56 \\
\hline
\end{tabular}

Aos 110 dias após o transplantio, mensurou-se a altura (H) e diâmetro do coleto (DC). Foram tomadas duas mudas por parcela, com base no diâmetro médio, para as análises destrutivas. O índice de verde foi avaliado por meio do medidor portátil de clorofila (SPAD - 502), e a área foliar (AF) foi determinada por meio do medidor 
LICOR-LI3000. O sistema radicular foi separado da parte aérea e submetido ao processo de lavagem para retirada do substrato.

Para determinação da massa seca da parte aérea (MSPA) e do sistema radicular (MSSR), ambos os compartimentos foram levados para estufa de circulação forçada de ar a $65^{\circ} \mathrm{C}$ por 72 horas. Em seguida, o material foi pesado. Para determinação do índice de qualidade de Dickson, foi utilizada a equação a seguir:

$$
\mathrm{IQD}=\frac{\mathrm{MST}}{\frac{\mathrm{H}}{\mathrm{DC}}}+(\mathrm{MSPA} / \mathrm{MSSR})
$$

Em que: IQD: índice de qualidade de Dickson; MST: massa seca total; H: altura das mudas; DC: diâmetro do coleto; MSPA: massa seca da parte aérea; MSSR: massa seca do sistema radicular.

Após a determinação da MSPA, as amostras do material vegetal foram moídas em moinho de facas, tipo Willey, e pesados $0,1 \mathrm{~g}$ em tubos de ensaio para determinação dos teores de nitrogênio $(\mathrm{N})$, fósforo $(\mathrm{P})$ e potássio $(\mathrm{K})$, e estimativa dos conteúdos. As amostras foram submetidas à oxidação por digestão sulfúrica $\left(\mathrm{H}_{2} \mathrm{O}_{4}\right.$ e $\left.\mathrm{H}_{2} \mathrm{O}_{2}\right)$ em sistema de digestão aberta. Em seguida, o $\mathrm{N}$ foi determinado por meio do método Nessler, o $\mathrm{P}$ com espectofotômetro (Specord 210) pelo método colorimétrico (azul de molibdato), e o K, determinado no fotômetro de emissão de chama.

Com relação à qualidade do torrão formado, avaliou-se agregação do torrão (AGRE), ou seja, a quantidade de substrato aderido ao sistema radicular, sendo atribuídas notas de 1 a 5, em que: nota 1 representa menos de $20 \%$ do substrato aderido ao sistema radicular; nota 2, de 20 a 40\%; nota 3, de 40 a $60 \%$; nota 4, de 60 a $80 \%$; e nota 5 , de 80 a $100 \%$. Para determinar a porcentagem aderida, foi pesado o torrão (raiz + substrato) e o que restou no tubete. Desse valor, foi descontado o valor da massa fresca de cada raiz, pesada após a lavagem, sendo então estimada a porcentagem de substrato aderido ao sistema radicular.

A curvatura do torrão (CURV) foi avaliada de acordo com o grau de deformação ao ser colocado suspenso na horizontal, atribuindo-se notas de 1 a 5 . Classificou-se com nota 1 , a partir de $25^{\circ}$ de curvatura ou quando o torrão apresentou rachaduras ou quebra; nota 2 , de $20-25^{\circ}$; nota 3 , de $15-20^{\circ}$; nota 4 , de $10-15^{\circ}$; e nota 5 , de $5-10^{\circ}$ de curvatura. Os dados obtidos foram submetidos à análise de variância (ANOVA) e comparação das diferenças pelo teste de Tukey ( $5 \%$ de probabilidade). As variáveis qualitativas foram comparadas pelo teste não paramétrico de Kruskal-Wallis (5\% de probabilidade).

\section{RESULTADOS}

As mudas produzidas em substratos que continham LET apresentaram superioridade em todas as características biométricas avaliadas em relação àquelas produzidas em SC puro (Tabela 2). A relação massa seca do sistema radicular/massa seca da parte aérea (MSSR/MSPA) indica a distribuição da biomassa entre o sistema radicular e a parte aérea. Neste trabalho, as mudas produzidas em substratos que continham LET apresentaram valores inferiores desta relação quando comparadas àquelas produzidas em SC puro.

Tabela 2. Altura (H), diâmetro do coleto (DC), área foliar (AF), massa seca da parte aérea (MSPA), massa seca do sistema radicular (MSSR) e relação massa seca do sistema radicular/massa seca da parte aérea (MSSR/MSPA) de mudas de Lafoensia glyptocarpa produzidas em diferentes substratos aos 120 dias após a semeadura.

Table 2. Height $(\mathrm{H})$, stem diameter (DC), leaf area (AF), shoot dry mass (MSPA), root dry mass (MSSR) and root dry mass/shoot dry mass ratio of Lafoensia glyptocarpa seedlings produced in different substrates at 120 days after sowing.

\begin{tabular}{ccccccc}
\hline \multirow{2}{*}{ Tratamento } & $\mathbf{H}$ & $\mathbf{D C}$ & $\mathbf{A F}$ & MSPA & MSSR & MSSR/MSPA \\
\cline { 2 - 6 } & $\mathbf{c m}$ & $\mathbf{m m}$ & $\mathbf{c m}^{\mathbf{2}}$ & $\mathbf{g}$ & $\mathbf{g}$ & $0,97 \mathrm{~b}$ \\
$0 \% \mathrm{LET}+\mathrm{SC}$ & $9,59 \mathrm{~b}^{\mathbf{1}}$ & $1,34 \mathrm{c}$ & $24,6 \mathrm{c}$ & $0,164 \mathrm{c}$ & $0,1466 \mathrm{~b}$ & $0,55 \mathrm{a}$ \\
$25 \% \mathrm{LET}+\mathrm{SC}$ & $21,54 \mathrm{a}$ & $2,7 \mathrm{a}$ & $107,2 \mathrm{~b}$ & $0,935 \mathrm{~b}$ & $0,5073 \mathrm{a}$ & $0,46 \mathrm{a}$ \\
$50 \%$ LET + SC & $20,58 \mathrm{a}$ & $2,4 \mathrm{ab}$ & $105,3 \mathrm{~b}$ & $0,903 \mathrm{~b}$ & $0,4234 \mathrm{a}$ & $0,39 \mathrm{a}$ \\
$75 \%$ LET + SC & $22,70 \mathrm{a}$ & $2,62 \mathrm{ab}$ & $157,8 \mathrm{a}$ & $1,254 \mathrm{a}$ & $0,4885 \mathrm{a}$ & $0,28 \mathrm{a}$ \\
$100 \%$ LET & $21,15 \mathrm{a}$ & $2,1 \mathrm{~b}$ & $167,5 \mathrm{a}$ & $1,451 \mathrm{a}$ & $0,4226 \mathrm{a}$ & 37,16 \\
\hline
\end{tabular}

${ }^{1}$ Médias seguidas de letras iguais na coluna não diferem entre si pelo teste de Tukey a 5\% de significância. 
Neste trabalho, as mudas produzidas em substratos que utilizaram o LET apresentaram mesmo IQD, independente da proporção utilizada, porém superiores àquelas produzidas em SC puro (Tabela 3). Assim como as variáveis biométricas, os maiores valores do índice de verde (SPAD) foram observados nas mudas produzidas em substratos com LET em relação àquelas produzidas em SC puro (Tabela 3).

Os menores conteúdos de nitrogênio $(\mathrm{N})$, fósforo $(\mathrm{P})$ e potássio $(\mathrm{K})$ das mudas foram observados naquelas produzidas em SC puro, sem adição de qualquer fertilização.

Tabela 3. Índice de qualidade de Dickson (IQD), índice de verde (SPAD), e conteúdo de nitrogênio (N), fósforo (P) e potássio (K) da parte aérea de mudas de Lafoensia glyptocarpa produzidas em diferentes substratos aos 120 dias após a semeadura.

Table 3. Dickson quality index (IQD), green index (SPAD) and nitrogen $(\mathrm{N})$, phosphorus $(\mathrm{P})$, and potassium $(\mathrm{K})$ content in shoot dry mass of Lafoensia glyptocarpa seedlings produced in different substrates at 120 days after sowing.

\begin{tabular}{|c|c|c|c|c|c|}
\hline Tratamento & IQD & SPAD & $\mathbf{N}$ & $\mathbf{P}$ & $\mathbf{K}$ \\
\hline & & & \multicolumn{3}{|c|}{ 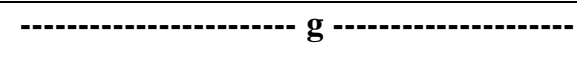 } \\
\hline $0 \% \mathrm{LET}+\mathrm{SC}$ & $0,04 b^{1}$ & $30,10 \mathrm{~b}$ & $2,38 \mathrm{~d}^{1}$ & $0,47 \mathrm{~b}$ & $2,03 \mathrm{c}$ \\
\hline $25 \%$ LET + SC & $0,14 \mathrm{a}$ & 40,66 a & $14,53 \mathrm{c}$ & $1,97 \mathrm{a}$ & $9,23 \mathrm{~b}$ \\
\hline $50 \%$ LET + SC & $0,12 \mathrm{a}$ & $33,16 \mathrm{~b}$ & $15,38 \mathrm{bc}$ & $2,05 \mathrm{a}$ & $11,54 \mathrm{ab}$ \\
\hline $75 \% \mathrm{LET}+\mathrm{SC}$ & $0,15 \mathrm{a}$ & 44,12 a & $21,75 \mathrm{ab}$ & $2,41 \mathrm{a}$ & $13,33 \mathrm{a}$ \\
\hline $100 \%$ LET & $0,14 \mathrm{a}$ & 45,70 a & 28,09 a & $2,59 \mathrm{a}$ & $13,58 \mathrm{a}$ \\
\hline $\mathrm{CV}(\%)$ & 16,27 & 7,47 & 21,04 & 21,03 & 18,10 \\
\hline
\end{tabular}

${ }^{1}$ Médias seguidas de letras iguais na coluna não diferem entre si pelo teste de Tukey a 5\% de significância.

Com relação à qualidade do torrão formado, pouco abordada na literatura, não houve efeito da adição do LET para a agregação do substrato ao torrão (Tabela 4).

Tabela 4. Pontuação qualitativa dada à agregação (AGRE) e curvatura do torrão (CURV) de mudas de Lafoensia glyptocarpa produzidas em diferentes substratos aos 120 dias após a semeadura.

Table 4. Qualitative score given for clod agregation (AGRE) and curvature (CURV) in Lafoensia glyptocarpa seedlings produced in different substrates at 120 days after sowing.

\begin{tabular}{ccc}
\hline Tratamento & AGRE & CURV \\
\hline 0\% LET + SC & 4,8 & $1,9 \mathrm{~b}^{1}$ \\
25\% LET + SC & 5,0 & $4,2 \mathrm{a}$ \\
$50 \%$ LET + SC & 4,7 & $3,7 \mathrm{ab}$ \\
$75 \%$ LET + SC & 5,0 & $3,2 \mathrm{ab}$ \\
100\% LET & 5,0 & $2,5 \mathrm{ab}$ \\
CV $(\%)$ & 6,61 & 38,24 \\
\hline
\end{tabular}

${ }^{1}$ Médias seguidas de letras iguais na coluna não diferem entre si pelo teste de Kruskal-Wallis a 5\% de significância.

Quanto à curvatura do torrão, as mudas produzidas em 25\% de LET + SC apresentaram torrão mais estável quando suspenso na horizontal em relação àquelas produzidas em substrato comercial puro.

\section{DISCUSSÃO}

Com relação ao crescimento das mudas de mirindiba-rosa, os resultados encontrados neste trabalho corroboram com o observado por Caldeira et al. (2013), Faria et al. (2013), Trazzi et al. (2014) e Cabreira et al. (2017), Isso indica que o lodo de esgoto na composição de substrato confere características superiores de crescimento às mudas, embora os autores acima afirmem que esse melhor desempenho possa não ocorrer em elevados percentuais do resíduo, o que não foi observado neste trabalho.

Trigueiro e Guerrini (2014) atribuíram os benefícios para o crescimento em altura de mudas de Schinus terebinthifolius à riqueza de nitrogênio presente no lodo de esgoto utilizado na composição do substrato. Santos $e t$ al. (2014) também afirmam que há elevação do teor de nitrogênio dos substratos que recebem o lodo de esgoto. Além do 
nitrogênio, o lodo possui elevados teores de outros nutrientes essenciais para as plantas, como fósforo, cálcio e micronutrientes (SANTOS et al., 2014), podendo ser o maior crescimento aéreo e radicular das mudas de Lafoensia glyptocarpa atribuído à tal riqueza nutricional, refletindo em maior AF, MSPA e MSSR (Tabela 2).

Ainda em relação ao crescimento, resultados divergentes aos deste trabalho foram verificados por Teles et al. (1999), que observaram redução em H e DC de mudas de Enterolobium contortisiliquum produzidas em diferentes proporções de LET e terra de subsolo. Para higienização e estabilização do resíduo, os autores também utilizaram a adição de cal. Entretanto, a aplicação foi feita a $50 \%$ do peso seco, superior à utilizada neste trabalho (15\%). A aplicação de cal eleva o pH do resíduo, o que pode influenciar negativamente o desenvolvimento das mudas (NOVAIS et al., 2007). Contudo, não foram observados prejuízos para o crescimento das mudas de mirindiba-rosa, mesmo quando utilizado $100 \%$ de LET na composição do substrato aos 120 após a semeadura.

Em relação à MSPA, resultados semelhantes aos deste trabalho foram obtidos por Scheer et al. (2012a), que avaliaram o uso do lodo de esgoto na produção de mudas de Anadenanthera colubrina. Os autores observaram que os tratamentos que receberam o resíduo apresentaram mudas com maior acúmulo de MSPA quando comparados ao substrato comercial puro.

Gomes e Paiva (2011) ressaltam que a sobrevivência, o estabelecimento e a boa produtividade dos povoamentos florestais são consideravelmente maiores quanto mais abundante for o sistema radicular, independente da altura da parte aérea, que pode ser representado pela MSSR. Assim, essa afirmação indica que, para este trabalho, as mudas que receberam LET têm maior probabilidade de sucesso pós plantio em relação àquelas produzidas em substrato comercial puro.

Para a relação matéria seca do sistema radicular/ matéria seca da parte aérea (MSSR/MSPA), dentre as proporções de LET utilizadas, as de 25 e $50 \%$ proporcionaram valores mais próximos ao valor de 0,5 , sugerido como ideal por Caldeira et al. (2008), elevando a probabilidade de sobrevivência pós plantio das mudas (CALDEIRA et al., 2013). Os maiores valores observados nas mudas produzidas em SC puro indicam que houve desbalanço do crescimento aéreo em relação ao radicular neste tratamento.

O índice de qualidade de Dickson (IQD) pode ser considerado como indicador da qualidade das mudas, sendo maior a qualidade da muda quanto maior o seu valor. Scheer et al. (2012b), ao avaliar o uso de lodo de esgoto em mudas de Lafoensia pacari, afirmaram que todos os tratamentos utilizando lodo proporcionaram maior IQD quando comparadas as mudas em substrato comercial, semelhante ao encontrado neste trabalho. Os mesmos autores ainda sugerem a fertilização química complementar, embora o lodo tenha apresentado ótimo resultado como substrato para produção das mudas.

Abreu et al. (2017), também avaliando a produção de mudas de Lafoensia pacari em lodo de esgoto, afirmaram que quando em mistura com $20 \%$ de fibra de coco, o lodo pode ser recomendado como substrato para essa espécie. Os resultados foram superiores em relação ao substrato comercial, assim como observado no presente trabalho.

Em relação ao índice de verde (SPAD), diretamente correlacionado com o teor de clorofila, os resultados observados validam a pressuposição do maior crescimento das mudas (Tabela 2) ter sido atribuído à riqueza nutricional do LET, em especial, o nitrogênio. Entretanto, na proporção de 50\% de LET, houve redução desse índice em comparação com as demais proporções de LET utilizadas.

$\mathrm{O}$ menor conteúdo de $\mathrm{N}, \mathrm{P}$ e $\mathrm{K}$ verificado nas mudas produzidas em SC puro reafirma a capacidade do LET de suprir as necessidades nutricionais das mudas de mirindiba-rosa, com maior absorção desses nutrientes. Resultados semelhantes aos deste trabalho foram observados por Trazzi et al. (2014), que encontraram maior conteúdo de N, P e K em mudas de Tectona grandis produzidas em substratos que receberam LET, sendo este resultado também atribuído à maior disponibilidade desses nutrientes. Cunha et al. (2006) observaram maior acúmulo de $\mathrm{N}$ nas mudas de Acacia sp. produzidas na maior proporção de LET (100\%), principalmente quando inoculadas com rizóbios, o que pode ter favorecido o conteúdo de $\mathrm{N}$ nas mudas devido à associação com as bactérias diazotróficas fixadoras de nitrogênio.

A melhor nota qualitativa dada para a curvatura do torrão das mudas produzidas em $25 \%$ de LET em substrato comercial está diretamente relacionada com a boa formação do sistema radicular, expresso em MSSR (Tabela 2), o que propicia melhor agregação do substrato, torna o torrão mais firme, facilita os processos de manuseio, transporte e plantio das mudas no campo, embora sem diferença estatística com as demais proporções de LET utilizadas. Além disso, Cabreira et al. (2017) destacam ainda que, quando utilizado o LET na composição do substrato para produção de mudas florestais, o peso das mudas formadas é diminuído, facilitando a logística de expedição para o campo.

FLORESTA, Curitiba, PR, v. 48, n. 2, p. 277-284, abr/jun. 2018

Siqueira. D. P. et.al.

ISSN eletrônico 1982-4688

DOI: $10.5380 /$ rf.v48 i 2.55795 


\section{CONCLUSÕES}

- A qualidade das mudas de mirindiba-rosa (Lafoensia glyptocarpa) foi positivamente influenciada pela adição de lodo de esgoto tratado ao substrato, indicando a viabilidade e adequação deste uso.

- Para produção de mudas dessa espécie, recomenda-se proporções entre 25 e $100 \%$ de lodo de esgoto tratado em mistura com substrato comercial.

\section{AGRADECIMENTOS}

bolsas de estudo.

\section{REFERÊNCIAS}

ABREU, A.H.M.; MARZOLA, L.B.; MELO, L.A.; LELES, P.S.S.; ABEL, E.L.S.; ALONSO, J.M. Urban solid waste in the production of Lafoensia pacari seedlings. Revista Brasileira de Engenharia Agrícola e Ambiental. v.21, n.2, p.83-87, 2017.

ABREU-HARBICH, L.V.; LABAKI, L.C.; MATZARAKIS, A. Effect of tree planting design and tree species on human thermal comfort in the tropics. Landscape and Urban Planning. V.138, P.99-109, 2015.

CABREIRA, G.V.; LELES, P.S.S.; ALONSO, J.M.; ABREU, A.H.M.; LOPES, N.F.; SANTOS, G.R. Biossólido como componente de substrato para produção de mudas florestais. Floresta, Curitiba, v.47, n.2, p.165-176, 2017

CALDEIRA, M.V.W.; PERONI, L.; GOMES, D.R.; DELARMELINA, W.M.; TRAZZI, P.A. Diferentes proporções de biossólido na composição de substratos para produção de muda de timbó (Ateleia glazoviana Baill). Scientia Forestalis, Piracicaba, v.40, p.15-22, 2012.

CALDEIRA, M.V.W.; ROSA, G.N.; FENILLI, T.A.B.; HARBS, R.M.P. Composto orgânico na produção de mudas de aroeira-vermelha. Scientia Agraria, Curitiba, v.9, p.27-33, 2008.

CALDEIRA, M.V.W.; DELARMELINA, W.M.; FARIA, J.C.T.; JUVANHOL, R.S. Substratos alternativos na produção de mudas de Chamaescrista desvauxii. Revista Árvore, v.37, n.1, p.31-39, 2013.

CARVALHO, P.E.R. Espécies arbóreas brasileiras. Brasília: Embrapa Informação Tecnológica. v.1. 2003, 1039p.

CONSELHO NACIONAL DO MEIO AMBIENTE (CONAMA). Resolução. Resolução no 375 de 29 de agosto de 2006. Define critérios e procedimentos para o uso agrícola de lodos de esgoto gerados em estações de tratamento de esgoto sanitário e seus produtos derivados. Diário Oficial da União, Brasília, DF, trinta de agosto 2006. Disponível em: <http://www.mma.gov.br/port/conama/res/res06/res37506.pdf> Acesso em: 15 agosto 2017.

CUNHA, A.M.; CUNHA, G.M.; SARMENTO, R.A.; CUNHA, G.M.; AMARAL, J.F.T. Efeito de diferentes substratos sobre o desenvolvimento de mudas de Acacia sp. Revista Árvore, Viçosa, v.30, n.2, p.207-214, 2006.

DELARMELINA, W.M.; CALDEIRA, M.V.W.; FARIA, J.C.T.; GONÇALVES, E.O.; ROCHA, R.L.F. Diferentes substratos para produção de mudas de Sesbania virgata. Floresta e Ambiente, Seropédica, v.21, p. 224-233, 2014.

FARIA, J.C.T.; CALDEIRA, M.V.W.; DELARMELINA, W.M.; GONÇALVES, E.O. Uso de resíduos orgânicos no crescimento de mudas de Mimosa setosa. Pesquisa Florestal Brasileira, v.33, n.76, p.409-418, 2013.

GOMES, D.R.; CALDEIRA, M.V.W.; DELARMELINA, W.M.; GONÇALVES, E.O.; TRAZZI, P.A. Lodo de esgoto como substrato para a produção de mudas de Tectona grandis L. Revista Cerne, v.19, n.1, p.123-131, 2013.

GOMES, J.M.; PAIVA, H.N. Viveiros florestais: propagação sexuada. Editora UFV - Viçosa, MG. Série didática, 2011, 116p.

LORENZI, H. Árvores brasileiras: manual de identificação e cultivo de plantas arbóreas nativas do Brasil. 2.ed. Nova Odessa: Instituto Plantarum. v.2. 2002, 382p. 
NOBREGA, M.A.S.; PONTES, M.S.; SANTIAGO, E.F. Incorporação do lodo de esgoto na composição de substrato para produção de mudas nativas. Acta Biomedica Brasiliensia. v.8, n.1, p.43-55, 2017.

NOVAIS, R.F.; VENEGAS, V.H.A.; BARROS, N.F.; FONTES, R.L.; CANTARUTTI, R.B.; NEVES, J.C.L. Fertilidade do solo. Viçosa, MG. Sociedade brasileira de ciência do solo, $1^{\text {a }}$ Ed. 2007, 1017p.

ROCHA, J.H.T.; BACKES, C.; DIOGO, F.A.; PASCOTTO, C.B.; BORELLI, K. Composto de lodo de esgoto como substrato para mudas de eucalipto. Pesquisa Florestal Brasileira,Curitiba. v.33, n.73, p.27-36, 2013.

SANTOS, E.V.F.; KUNZ, S.H.; CALDEIRA, M.V.W.; AZEVEDO, C.H.S.; RANGEL, O.J.P. Características químicas de substratos formulados com lodo de esgoto para produção de mudas florestais. Revista Brasileira de Engenharia Agrícola e Ambiental. Campina Grande, v.18, p.971-979, 2014.

SCHEER, M.B.; CARNEIRO, C.; BRESSAN, A.O.; SANTOS, K.G. Composto de lodo de esgoto para a produção de mudas de Anadenanthera colubria (Vell.) Brenan. Cerne, Lavras, v.18, n.4, p.613-621, $2012 \mathrm{a}$.

SCHEER, M.B; CARNEIRO, C., BRESSAN, O.A.; SANTOS, K.G. Crescimento e nutrição de mudas de Lafoensia pacari com lodo de esgoto. Floresta e Ambiente. Seropédica, v.19, p.55-65, 2012 b.

TELES, C.R.; COSTA, A.N.; GONÇALVES, R.F. Produção de lodo em lagoas de estabilização e seu uso no cultivo de espécies florestais na região Sudoeste do Brasil. Sanare, Vitória, v.12, p.1-12, 1999.

TRAZZI, P.A.; CALDEIRA, M.V.W.; REIS, E.F.; SILVA, A.G. Produção de mudas de Tectona grandis em substratos formulados com biossólido. Cerne, Lavras, v.20, p.293-302, 2014.

TRIGUEIRO, R.M.; GUERRINI, I.A. Utilização de lodo de esgoto na produção de mudas de Aroreira-pimenteira. Revista Árvore, Viçosa, v.38, n.4, p.657-665, 2014. 
FLORESTA, Curitiba, PR, v. 48, n. 2, p. 277-284, abr/jun.2108

Siqueira. D. P. et.al 